\title{
Hexagulation numbers: the magic numbers of equal spheres on triply periodic minimal surfaces
}

\author{
Tomonari Dotera $^{1} \cdot$ Hideaki Tanaka ${ }^{1} \cdot$ Yusuke Takahashi $^{1}$
}

Received: 9 July 2016/ Accepted: 20 August 2016/Published online: 2 September 2016

(c) The Author(s) 2016. This article is published with open access at Springerlink.com

\begin{abstract}
Regular structures of equal spheres on the triply periodic minimal surfaces known as primitive $(\mathrm{P})$, gyroid (G) and diamond (D) surfaces are enumerated as obtained through Monte Carlo simulations of hard spheres undergoing the Alder transition. Remarkably, there exist magic numbers producing the regular structures, which are simply explained by means of hexagulation numbers defined as $H=h^{2}+k^{2}-h k$, in analogy with the Caspar and Klug's triangulation numbers, $T=h^{2}+k^{2}+h k$ for icosahedral viruses, where $h$ and $k$ are equal to nonnegative integers. Understanding the significance of symmetry of the surfaces, the total number of spheres per cubic unit cell $N$ is represented by $N=8 H, 16 H$, and $32 H$ for P-, G- and $\mathrm{D}$-surfaces, respectively. Accordingly, these arrangements are analyzed in terms of space groups, equivalent positions (Wyckoff positions), and polygonal-tiling representations. The key is that there is only a limited number of efficient physical design possible even on the triply periodic minimal surfaces.
\end{abstract}

Keywords Triply periodic minimal surfaces · Gyroid surface $\cdot$ Triangulation number $\cdot$ Hard spheres $\cdot$ Alder transition $\cdot$ Monte Carlo simulation

Dedicated to Professor Alan Mackay on the occasion of his 90th birthday.

Tomonari Dotera

dotera@phys.kindai.ac.jp

1 Department of Physics, Kindai University, Kowakae 3-4-1, Higashi-Osaka 577-8502, Japan

\section{Introduction}

On a flat surface, the hexagonal arrangement is a ubiquitous regular arrangement of spherical particles, arising from dense packing, space division, entropic ordering, or interactions between particles [1]. What is the regular arrangement of particles when the surface is curved? On a spherical surface, this question was firstly raised by Thomson [2], followed by Fejes Tóth [3] and Mackay et al. [4]. For regular arrangements in particular, Goldberg [5] elucidated regular polyhedra consisting of pentagonal and hexagonal faces whose centers correspond to the positions of particles, and later for biological icosahedral viruses, Caspar and Klug proposed a construction principle of regular arrangements inspired by Buckminster Fuller's geodesic dome [6-8].

In contrast, regular arrangements on a saddle-shaped surface with negative Gaussian curvature have yet to be fully elucidated [9-14]. Mackay and Terrones [15] proposed a periodic tiling on the primitive surface inspired by fullerenes, and several tilings have been investigated from mathematical viewpoints [16, 17], and furthermore, a large number of 3D networks have been enumerated [18, 19]; however, physical interactions have not been taken into consideration. In this paper, we explore crystalline phases on triply periodic minimal surfaces (TPMSs) using Monte Carlo (MC) simulations of hard spheres. The TPMSs we consider here are the most famous three, namely the Schwarz primitive (P) and diamond (D) surfaces, and the Schoen gyroid $(G)$ surface, which are abundant in various soft materials such as biological lipids, butterfly wings, surfactants, mesoporous materials, and block copolymers [20-29].

It is well known that spherical virus capsids employ efficient designs using the icosahedral symmetry, leading 
to the regularity of the numbers of subunits on the spherical surface, which was explained by Caspar and Klug using the concept of the triangulation number $T$ [6, 7]. Surprisingly, we have found only discrete numbers undergoing clear fluid-solid transitions in our MC simulations for the TPMSs. For cubic symmetry in particular, we unravel these magic numbers in terms of a similar number, the hexagulation number $H$, which is the main result of the present paper. Future applications of such regular arrangements are to construct complex membranes using the concepts of bijels, colloidosomes, and polymersomes [30-33].

The paper is organized as follows: Section "Monte Carlo simulations" briefly explains the Monte Carlo method and presents results of simulations. We list the magic numbers and their space group symmetries and polygonal tilings. All coordinates of regular arrangements are provided in terms of Wyckoff positions of the space groups. In "Hexagulation Number," we first review the triangulation number and then define the hexagulation number to explain the magic numbers on TPMSs. Here, we will focus regular arrangements with original space groups of TPMSs or their cubic subgroups exhibiting a strong similarity with the icosahedral viruses. Finally, discussion is given in the last section.

\section{Monte Carlo simulations}

It is widely known that even a purely hard-sphere system undergoes a fluid-solid transition, called the Alder transition, producing the hexagonal arrangement [34], whose entropy-driven ordering mechanism turns out to be universal in soft materials [35]. Above a certain critical density, the solid phase in the hexagonal arrangement gains more entropy than the fluid phase. Do regular arrangements emerge in the solid phase on the TPMSs?

Motivated by this possibility, we began an investigation on the G-surface and indeed found the Alder transition, but only for specific numbers of spheres [14]. To provide further insight into the regular arrangement and establish a unified framework, we have extended our MC simulations to the P- and D-surfaces in this study.

The well-known approximate forms $[36,37]$ of the surfaces are described as

$$
\begin{aligned}
P: s(\mathbf{q})= & \cos x+\cos y+\cos z=0, \\
G: s(\mathbf{q})= & \sin x \cos y+\sin y \cos z+\sin z \cos x=0, \\
D: s(\mathbf{q})= & \sin x \sin y \sin z+\sin x \cos y \cos z \\
& +\cos x \sin y \cos z+\cos x \cos y \sin z=0,
\end{aligned}
$$

where $\mathbf{q}=(x, y, z)$ and the lattice constant is taken to be unity; $2 \pi$ is omitted in the expressions.
In practice, the centers of spheres are confined between boundaries $s(\mathbf{q})=-0.1$ (green) and $s(\mathbf{q})=0.1$ (red) as shown in Fig. 1a-c. We assign $N_{\alpha}$ to the number of spheres per cubic unit cell of the surfaces. $\alpha$ stands for $P, G$, and $D$. We begin with a random state having a sufficiently small radius of spheres on the TPMSs, perform a MC run without any symmetry input, then increase the radius $r$ with a small increment, and repeat the process. Details of the method of the simulations were given in Ref. [14].

The order parameter $h(r)$ as a function of sphere radius $r$ is defined as

$$
h(r)=\left\langle\frac{1}{M} \sum_{i=1}^{M} f_{h k l}\left(\mathbf{q}_{i}\right)\right\rangle,
$$

where the sum is taken over positions of all sphere centers $\mathbf{q}_{i}$, and $\langle\cdots\rangle$ implies the MC average. The function $f_{h k l}(\mathbf{q})$ is an invariant function under the operations of the space groups, ex. $\operatorname{Im} \overline{3} m$ for the P-surface [38]:

$f_{h k l}(\mathbf{q})=\sum_{\mathbf{q} \in \mathbf{C}_{3}} \cos (h x) \cos (k y) \cos (l z)$,

where $(h, k, l)$ is a set of integers and $C_{3}$ is a group of all cyclic permutations of $(x, y, z)$. Suitable choices of $(h, k, l)$ for each $N_{\alpha}$ give large absolute values of $h(r)$ in the ordered phase.

Figure 1d shows a snapshot of an ordered structure for a $N_{\mathrm{P}}=72$ system on the P-surface, in which alignments are observed in $x, y$, and $z$ directions despite undulations. To find clear phase behavior, we have employed simulation boxes consisting of multiple cells, here for instance, $3 \times$ $3 \times 2$ cells with periodic boundary conditions; accordingly, the number of spheres is 1296 for $N_{\mathrm{P}}=72$. The existence of the fluid-solid transition is judged firstly by whether there exists a discontinuous jump or not in the acceptance ratio (AR) curve representing the movable probability of $\mathrm{MC}$ trial moves of spheres. If not, the simulation ends up with a frozen random state with a continuous single curve. For $N_{\mathrm{P}}=72$, Fig. 1e exhibits discontinuous jumps and accordingly a hysteresis loop, indicating clear evidence of the first-order transition. Similarly, in Fig. 1f, the order parameter $h(r)$ is plotted, where $f_{045}(\mathbf{q})$ is chosen in Eq. (1). The transition points are exactly the same as those in Fig. 1e, implying that the ordered phase near the transition region has more movable spaces for spheres than in the disordered phase, and the ordered phase is, therefore, a high entropy phase. We have examined several system sizes to check the results.

Our observation indicates that the number of spheres in a unit cell having a definite fluid-solid transition shows discrete integers. For the P-surface, we find magic numbers $N_{\mathrm{P}}=20,32,56,72$, and 96; for the G-surface, we find $N_{\mathrm{G}}=40,48,64,112$, and 144; and for the D-surface, 
Fig. 1 Triply periodic minimal surfaces: a P-surface; b Gsurface; c D-surface. d Snapshot of self-organized spheres with radii $r=0.089$ on the $\mathrm{P}$-surface for a $N_{\mathrm{P}}=72$ system, where $N_{\mathrm{P}}$ is the number of spheres in a unit cell. Plots of $\mathbf{e}$ the acceptance ratio (AR) and $\mathbf{f}$ an order parameter $h(r)$ for the $N_{\mathrm{P}}=72$ system as functions of sphere radius: Solid line, up; dashed line, down (Color figure online) (a)

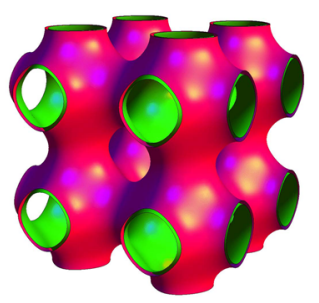

(b)

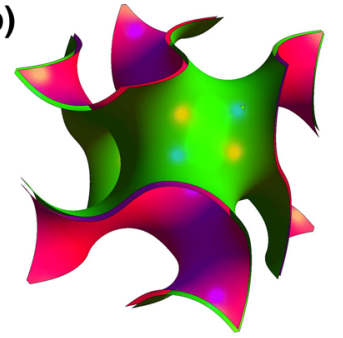

(c)

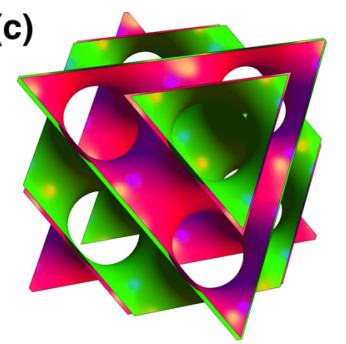

(d)

(e)
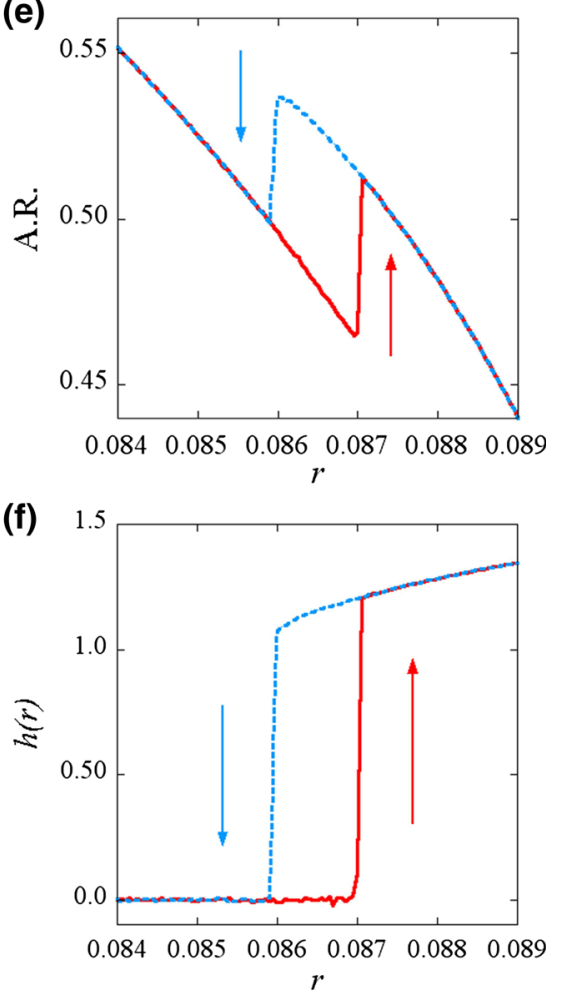

$N_{\mathrm{D}}=96,128$, and 224 are obtained. They can be best classified according to the hexagulation numbers $H$, which will be discussed in the next section.

Table 1 summarizes regular structures obtained from MC simulations, where we focus our attention on structures with cubic symmetry, since the TPMSs have inherent cubic space group symmetries, $\operatorname{Im} \overline{3} m, \operatorname{Ia} \overline{3} d$, and $P n \overline{3} m$ for P-, G- and D-surfaces, respectively. Several symmetry operations of the original space groups are broken in some cases, and the ordered arrangements are accordingly assigned to subgroups. For the $N_{\mathrm{P}}=72$ system, for instance, the arrangement is assigned to the space group $\operatorname{Pm} \overline{3} n \subset \operatorname{Im} \overline{3} m$. We should mention that the $\overline{H=4}$ class denotes hypothetical structures, while the $H=4$ class is obtained from simulations. They are very similar and the difference of the positions on the surfaces is very small, but the $\overline{H=4}$ class is more symmetric than that of $H=4$. Because the distances between spheres are more equivalent in $H=4$, and consequently in simulations, the symmetry of $\overline{H=4}$ is broken into the entropically more favorable $H=4$.

For eager readers, in Table 2 we explicitly provide the values of coordinates of spheres obtained by our MC simulations. One can consult Wyckoff positions in International Tables for Crystallography and evaluate all coordinates. Note that the Wyckoff position $96 \mathrm{~h}$ of $F \overline{4} 3 c$ for $N_{\mathrm{D}}=96$ in the International Tables is not convenient because of the different choices of the origin for symmetry operation. A part of $192 \mathrm{~h}$ of $F d \overline{3} c$ is more useful to reproduce our data, which are presented in Table 3.

Polygonal tilings are constructed from the center positions of spheres, represented in Fig. 2a-i. Since the magic numbers are larger for the G- and D-surfaces, we render five results for the P-surface and two for the G- and Dsurfaces. The set of integers $\left(n_{1}, n_{2}, n_{3}, \ldots ; n_{4}, n_{5}, n_{6}, \ldots ;\right)$ denotes a tiling of multiple vertex types in the way that $n_{1}$ gon, $n_{2}$-gon, and $n_{3}$-gon, $\cdots$ meet consecutively on each vertex, and superscripts are employed to abbreviate when 
Table 1 Regular tessellations of hard spheres on the P-, G-, and D-surfaces, classified by the hexagulation number $H$, the total number of spheres in a unit cell $N_{\alpha}$
Table 2 Explicit coordinates in terms of Wyckoff positions for the regular tessellations shown in Table 1

\begin{tabular}{|c|c|c|c|c|}
\hline H-number & Surface $\left(N_{\alpha}\right)$ & Space group & Wyckoff p. & Tiling \\
\hline \multirow[t]{2}{*}{$H^{*}=3$} & $\mathrm{P}(20)$ & $\operatorname{Im} \overline{3} m$ (No. 229) & $8 \mathrm{c} ; 12 \mathrm{~d}$ & $\left(3^{6} ; 3^{8}\right)$ \\
\hline & $\mathrm{G}(40)$ & $I a \overline{3} d$ (No. 230) & $16 \mathrm{a} ; 24 \mathrm{~d}$ & $\left(3^{6} ; 3^{8}\right)$ \\
\hline \multirow[t]{2}{*}{$H=3$} & G (48) & $I \overline{4} 3 d$ (No. 220) & $48 \mathrm{e}$ & $\left(3^{3} \cdot 4.3 .4\right)$ \\
\hline & $\mathrm{D}(96)$ & $F \overline{4} 3 c$ (No. 219) & $96 \mathrm{~h}$ & $\left(3^{3} \cdot 4.3 .4\right)$ \\
\hline \multirow[t]{3}{*}{$H=4$} & $\mathrm{P}(32)$ & $P n \overline{3}$ (No. 201) & $4 b ; 4 c ; 24 h$ & $\left(3^{6} ; 3^{7}\right)$ \\
\hline & G (64) & $I a \overline{3}$ (No. 206) & $8 \mathrm{a} ; 8 \mathrm{~b} ; 48 \mathrm{e}$ & $\left(3^{6} ; 3^{7}\right)$ \\
\hline & D (128) & $F d \overline{3}$ (No. 203) & $16 \mathrm{c} ; 16 \mathrm{~d} ; 96 \mathrm{~g}$ & $\left(3^{6} ; 3^{7}\right)$ \\
\hline \multirow[t]{3}{*}{$\overline{H=4}$} & $P(32)$ & $\operatorname{Im} \overline{3} m$ (No. 229) & $8 \mathrm{c} ; 24 \mathrm{~h}$ & $\left(3^{6} ; 3^{2} \cdot 4.3^{2} \cdot 4\right)$ \\
\hline & G (64) & $I a \overline{3} d$ (No. 230) & $16 \mathrm{a} ; 48 \mathrm{~g}$ & $\left(3^{6} ; 3^{2} \cdot 4 \cdot 3^{2} \cdot 4\right)$ \\
\hline & D (128) & $F d \overline{3} c$ (No. 228) & $32 \mathrm{c} ; 96 \mathrm{~g}$ & $\left(3^{6} ; 3^{2} \cdot 4 \cdot 3^{2} \cdot 4\right)$ \\
\hline \multirow[t]{3}{*}{$H=7$} & $\mathrm{P}(56)$ & $\operatorname{Pn} \overline{3} n$ (No. 222) & $8 \mathrm{c} ; 48 \mathrm{i}$ & $\left(3^{6} ; 3^{5} \cdot 4\right)$ \\
\hline & G (112) & $I a \overline{3} d$ (No. 230) & $16 a ; 96 h$ & $\left(3^{6} ; 3^{5} \cdot 4\right)$ \\
\hline & D (224) & $F d \overline{3} c$ (No. 228) & $32 \mathrm{c} ; 192 \mathrm{~h}$ & $\left(3^{6} ; 3^{5} \cdot 4\right)$ \\
\hline \multirow[t]{2}{*}{$H=9$} & $\mathrm{P}(72)$ & $\operatorname{Pm} \overline{3} n$ (No. 223) & $24 \mathrm{j} \times 2 ; 24 \mathrm{k}$ & $\left(3^{6} ; 3^{5} \cdot 4 ; 3^{5} \cdot 4\right)$ \\
\hline & G (144) & $I \overline{4} 3 d$ (No. 220) & $48 \mathrm{e} \times 3$ & $\left(3^{6} ; 3^{5} \cdot 4 ; 3^{5} \cdot 4\right)$ \\
\hline$H=12$ & $\mathrm{P}(96)$ & $I \overline{4} 3 m$ (No. 217) & $24 \mathrm{~g} \times 2 ; 48 \mathrm{~h}$ & $\left(3^{6} ; 3^{6} ; 3^{5} \cdot 4\right)$ \\
\hline
\end{tabular}

Notice that $N_{\mathrm{P}}=8 H, N_{\mathrm{G}}=16 H$ and $N_{\mathrm{D}}=32 \mathrm{H}$, and subscripts stand for the P-, G-, and D-surfaces. Space groups of the ordered structures are presented. Wyckoff positions are denoted as $m \mathrm{w}$, representing multiplicity $m$ and Wyckoff letter w corresponding to the space groups and polygonal tilings indicated by vertex. Some remarks: 1) Special cases in which spheres occupy the vertices of hexagonal patches, then we replace $H$ with $H=H^{*}-1 / 2$. 2) $\overline{H=4}$ class illustrates the hypothetical high-symmetry structures explained in the text. 3) P (32) with $P n \overline{3}$ is slightly broken from high symmetry $\operatorname{Im} \overline{3}$

\begin{tabular}{|c|c|c|c|c|c|c|}
\hline H-number & Surface $\left(N_{\alpha}\right)$ & Space group & Wyckoff p. & $x$ & $y$ & $z$ \\
\hline \multirow[t]{2}{*}{$H=3$} & G (48) & $I \overline{4} 3 d$ (No. 220$)$ & $48 \mathrm{e}$ & 0.576 & 0.386 & 0.554 \\
\hline & $\mathrm{D}(96)$ & $F \overline{4} 3 c$ (No. 219) & $96 h^{*}$ & 0.583 & 0.417 & 0.501 \\
\hline \multirow[t]{3}{*}{$H=4$} & $\mathrm{P}(32)$ & $P n \overline{3}$ (No. 201) & $24 \mathrm{~h}$ & 0.002 & 0.656 & 0.323 \\
\hline & $\mathrm{G}(64)$ & Ia $\overline{3}$ (No. 206) & $48 \mathrm{e}$ & 0.067 & 0.655 & 0.147 \\
\hline & D (128) & $F d \overline{3}$ (No. 203) & $96 \mathrm{~g}$ & 0.038 & 0.375 & 0.126 \\
\hline \multirow[t]{3}{*}{$H=7$} & $\mathrm{P}(56)$ & $P n \overline{3} n$ (No. 222) & $48 \mathrm{i}$ & 0.080 & 0.380 & 0.727 \\
\hline & G (112) & $I a \overline{3} d$ (No. 230) & $96 \mathrm{~h}$ & 0.039 & 0.144 & 0.411 \\
\hline & D (224) & $F d \overline{3} c$ (No. 228) & $192 \mathrm{~h}$ & 0.047 & 0.171 & 0.350 \\
\hline \multirow[t]{6}{*}{$H=9$} & $\mathrm{P}(72)$ & $P m \overline{3} n$ (No. 223) & $24 \mathrm{j}$ & - & 0.405 & - \\
\hline & & & $24 \mathrm{j}$ & - & 0.173 & - \\
\hline & & & $24 \mathrm{k}$ & - & 0.378 & 0.705 \\
\hline & G (144) & $I \overline{4} 3 d$ (No. 220$)$ & $48 \mathrm{e}$ & 0.011 & 0.064 & 0.550 \\
\hline & & & $48 \mathrm{e}$ & 0.010 & 0.650 & 0.604 \\
\hline & & & $48 \mathrm{e}$ & 0.064 & 0.339 & 0.355 \\
\hline \multirow[t]{3}{*}{$H=12$} & $\mathrm{P}(96)$ & $I \overline{4} 3 m$ (No. 217) & $24 \mathrm{~g}$ & 0.180 & - & 0.589 \\
\hline & & & $24 \mathrm{~g}$ & 0.212 & - & 0.329 \\
\hline & & & $48 \mathrm{~h}$ & 0.104 & 0.454 & 0.224 \\
\hline
\end{tabular}

Note that special points having exact values given in International Tables for Crystallography are omitted. For $96 \mathrm{~h}^{*}$ of $F \overline{4} 3 c$, see Wyckoff positions in Table 3 
Table 3 Wyckoff positions for $96 \mathrm{~h}^{*}$ of $F \overline{4} 3 c$ (No. 219) for $N_{\mathrm{D}}=96$

\begin{tabular}{llll}
\hline Coordinates & $+(0,0,0)+\left(0, \frac{1}{2}, \frac{1}{2}\right)$ & $+\left(\frac{1}{2}, 0, \frac{1}{2}\right)+\left(\frac{1}{2}, \frac{1}{2}, 0\right)$ & \\
\hline$(x, y, z)$ & $\left(\bar{x}+\frac{1}{4}, \bar{y}+\frac{3}{4}, z+\frac{1}{2}\right)$ & $\left(\bar{x}+\frac{3}{4}, y+\frac{1}{2}, \bar{z}+\frac{1}{4}\right)$ & $\left(x+\frac{1}{2}, \bar{y}+\frac{1}{4}, \bar{z}+\frac{3}{4}\right)$ \\
$(z, x, y)$ & $\left(z+\frac{1}{2}, \bar{x}+\frac{1}{4}, \bar{y}+\frac{3}{4}\right)$ & $\left(\bar{z}+\frac{1}{4}, \bar{x}+\frac{3}{4}, y+\frac{1}{2}\right)$ & $\left(\bar{z}+\frac{3}{4}, x+\frac{1}{2}, \bar{y}+\frac{1}{4}\right)$ \\
$(y, z, x)$ & $\left(\bar{y}+\frac{3}{4}, z+\frac{1}{2}, \bar{x}+\frac{1}{4}\right)$ & $\left(y+\frac{1}{2}, \bar{z}+\frac{1}{4}, \bar{x}+\frac{3}{4}\right)$ & $\left(\bar{y}+\frac{1}{4}, \bar{z}+\frac{3}{4}, x+\frac{1}{2}\right)$ \\
$\left(\bar{y}+\frac{1}{4}, \bar{x}+\frac{3}{4}, z\right)$ & $\left(y+\frac{1}{2}, x+\frac{1}{2}, z+\frac{1}{2}\right)$ & $\left(\bar{y}+\frac{3}{4}, x, \bar{z}+\frac{1}{4}\right)$ & $\left(y, \bar{x}+\frac{1}{4}, \bar{z}+\frac{3}{4}\right)$ \\
$\left(\bar{x}+\frac{1}{4}, \bar{z}+\frac{3}{4}, y\right)$ & $\left(x, \bar{z}+\frac{1}{4}, \bar{y}+\frac{3}{4}\right)$ & $\left(x+\frac{1}{2}, z+\frac{1}{2}, y+\frac{1}{2}\right)$ & $\left(\bar{x}+\frac{3}{4}, z, \bar{y}+\frac{1}{4}\right)$ \\
$\left(\bar{z}+\frac{1}{4}, \bar{y}+\frac{3}{4}, x\right)$ & $\left(\bar{z}+\frac{3}{4}, y, \bar{x}+\frac{1}{4}\right)$ & $\left(z, \bar{y}+\frac{1}{4}, \bar{x}+\frac{3}{4}\right)$ & $\left(z+\frac{1}{2}, y+\frac{1}{2}, x+\frac{1}{2}\right)$ \\
\hline
\end{tabular}

This is different from usual presentation in International Tables for Crystallography due to the different choice of the origin for symmetry operation. A part of $192 \mathrm{~h}$ of $F d \overline{3} c$ shown here is more useful to reproduce our MC data. Coordinates are $x=0.583, y=0.417$, and $z=0.501$
Fig. 2 Polygonal tiling connecting sphere centers. $N_{\alpha}$ is the number of vertices (spheres) per cubic unit cell, and subscripts, $\mathrm{P}, \mathrm{G}$, and $\mathrm{D}$ stand for the P-, G- and D-surfaces, respectively. $H^{*}=3, H=9$, and $H=12$ produce a $\left(3^{6} ; 3^{8}\right)$ tiling with $N_{\mathrm{P}}=20$, b $\left(3^{6} ; 3^{5} .4\right.$; $\left.3^{5} .4\right)$ tiling with $N_{\mathrm{P}}=72$, c $\left(3^{6}\right.$; $\left.3^{6} ; 3^{5} .4\right)$ tiling with $N_{\mathrm{P}}=96$, respectivley; $H=4$ generates $\left(3^{6} ; 3^{7}\right)$ tilings with $\mathbf{d} N_{\mathrm{P}}=32$, e $N_{\mathrm{G}}=64$, and $\mathbf{f} N_{\mathrm{D}}=128$; $H=7$ generates $\left(3^{6} ; 3^{5} .4\right)$ tilings with $\mathbf{g} N_{\mathrm{P}}=56, \mathbf{h}$ $N_{\mathrm{G}}=112$, and $\mathbf{i} N_{\mathrm{D}}=224$. Double cubic unit cells are rendered for the $\mathrm{P}$ - and G-surfaces, while a single cubic unit cell is rendered for the D-surface (Color figure online) (a) $N_{\mathrm{P}}=20\left(H^{*}=3\right)$

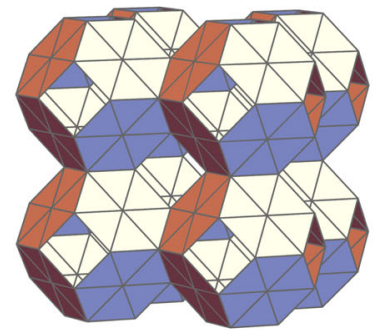

$\left(3^{6} ; 3^{8}\right)$

(d) $N_{\mathrm{P}}=32(H=4)$

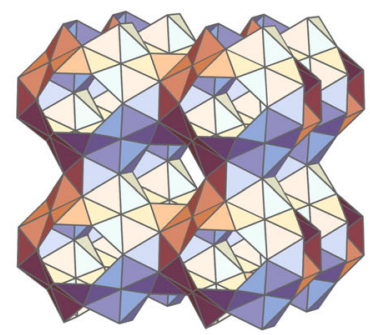

$\left(3^{6} ; 3^{7}\right)$

(g) $N_{\mathrm{P}}=56(H=7)$

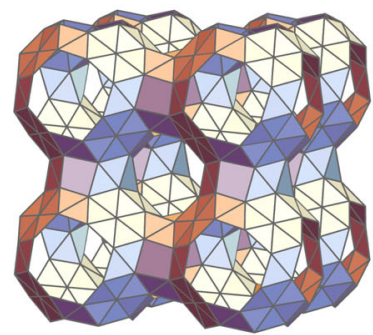

$\left(3^{6} ; 3^{5} .4\right)$ (b) $N_{\mathrm{P}}=72(H=9)$

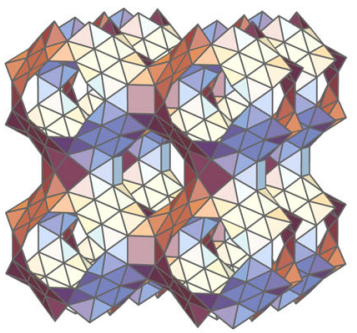

$\left(3^{6} ; 3^{5} .4 ; 3^{5} .4\right)$

(e) $N_{\mathrm{G}}=64(H=4)$

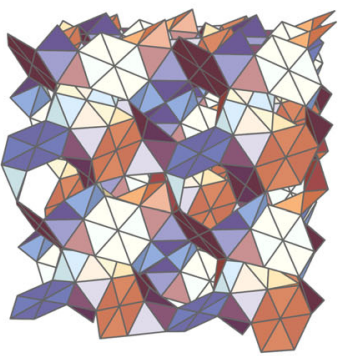

$\left(3^{6} ; 3^{7}\right)$

(h) $N_{\mathrm{G}}=112(H=7)$

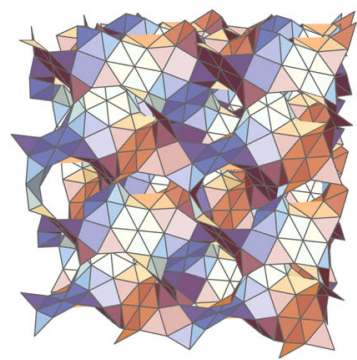

$\left(3^{6} ; 3^{5} \cdot 4\right)$ (c) $N_{\mathrm{P}}=96(H=12)$

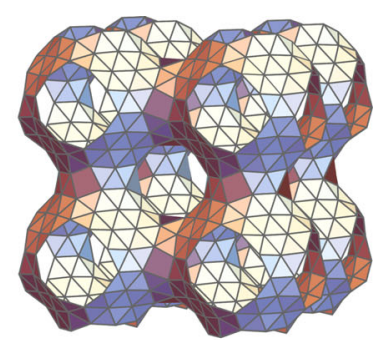

$\left(3^{6} ; 3^{6} ; 3^{5} .4\right)$

(f) $N_{\mathrm{D}}=128(H=4)$

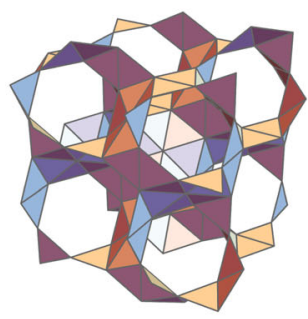

$\left(3^{6} ; 3^{7}\right)$

(i) $N_{\mathrm{D}}=224(H=7)$

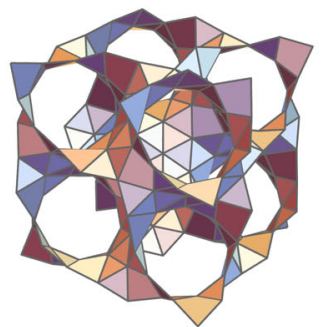

$\left(3^{6} ; 3^{5} .4\right)$ 
possible. A set of integers like $\left(3^{6} ; 3^{7}\right)$ denotes a tiling composed of two vertex types $3^{6}$ and $3^{7}$, for instance.

The tilings are composed of triangles and quadrangles, where length of all sides is almost equal within a few percent and where quadrangles are not always flat. Unlike the hexagonal arrangement $\left(3^{6}\right)$ on a flat plane, it should be noticed that vertices are not equivalent, as is the cases with icosahedral viruses. The first number of the Wyckoff positions listed in Table 1 represents the multiplicity of corresponding vertex types. All these tilings whose Euler characteristic $\chi$ per cubic unit cell is $-4,-8$, and -16 for P-, G-, and D-surfaces, respectively, are hyperbolic extensions of the flat plane tessellation $\left(3^{6}\right)$ with $\chi=0$. We finally point out strong similarity for the same $H$ classes as shown in Fig. $2 \mathrm{~d}-\mathrm{f}(H=4)$ and Fig. $2 \mathrm{~g}-\mathrm{i}(H=7)$.

\section{Hexagulation number}

Caspar and Klug showed that icosahedral shells can be constructed from twenty triangles with the triangulation number $T$ adopting the particular integer values $1,3,4,7,9,12, \ldots$, described by

$T=h^{2}+k^{2}+h k$

with $h, k$ equal to nonnegative integers. See Fig. 3a displaying $T$-diagram. A lattice point denoted as $(h, k)$ $\left(\mathbf{L}=h \mathbf{e}_{1}+k \mathbf{e}_{2}\right)$ in the oblique coordinate system spanned by basis vectors $\mathbf{e}_{1}$ and $\mathbf{e}_{2}$ on the triangular lattice indicates circled numbers $T$ defined as half the number of lattice points (or equivalently the number of triangles) inside the triangle whose side connects the origin and the point $(h, k)$. Considering the large triangle indicated by the solid line

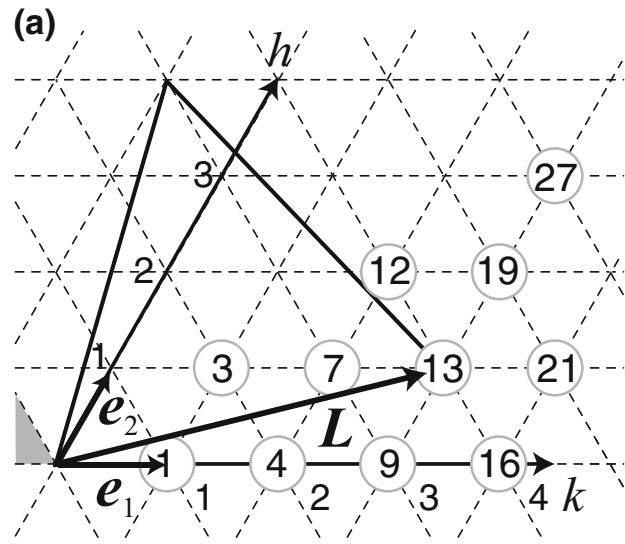

Fig. 3 a $T$-diagram for counting the triangulation number $T$ and $\mathbf{b} H$ diagram for counting the hexagulation number $H$. Lattice points denoted as $(h, k)\left(\mathbf{L}=h \mathbf{e}_{1}+k \mathbf{e}_{2}\right)$ with $h, k$ nonnegative integers in two oblique coordinate systems indicate circled numbers $T=h^{2}+$ and points occupying the vertices of the triangle in particular, the number of spheres $N$ for the total shell is given by $N=10 T+2$.

By the same token, we define the hexagulation number $H$ as the number of dashed hexagons inside a large hexagon (solid line) on $H$-diagram as shown in Fig. 3b. Let $\mathbf{L}=$ $h \mathbf{e}_{1}+k \mathbf{e}_{2}$ be a vector along an edge of the large hexagon, where $\mathbf{e}_{1}$ and $\mathbf{e}_{2}$ are basis vectors of the oblique coordinate system. Then, we can easily derive $H=|\mathbf{L}|^{2}$. Therefore, we have

$H=h^{2}+k^{2}-h k$,

generating $1,3,4,7,9,12, \ldots$, from sets of integers $(h, k)$. Note that the difference between $T$ and $H$ is just the plus or minus sign of the third term.

According to the space group symmetries, the TPMSs can be constructed from 8, 16, and 32 hexagonal patches per conventional cubic unit cell for P-, G-, and D-surfaces, respectively. Therefore, $N_{\alpha}$ is given by

$N_{\mathrm{P}}=8 H, \quad N_{\mathrm{G}}=16 H, \quad$ or,$\quad N_{\mathrm{D}}=32 H$

for the P-, G-, or D-surfaces, respectively. The regular structures listed in Table 1 obey these equations. For the special case described by $H^{*}=3$ in which spheres occupy the vertices of hexagonal patches, we should replace $H$ by $H^{*}-1 / 2$ in Eq. (3) as shown in Table 1.

It has been discussed in the literature that hyperbolic geometries on the Poincare disk can be conformally mapped upon the TPMSs $[18,21,39]$. See Fig. 4a-d. The dodecagonal region within twelve thick curves in Fig. 4b, d covers the area of a unit cell, a half, and one-fourth areas of a cubic unit cell of the P-, G-, and D-surfaces, respectively. The relation between the hexagon on the $H$-diagram and

(b)

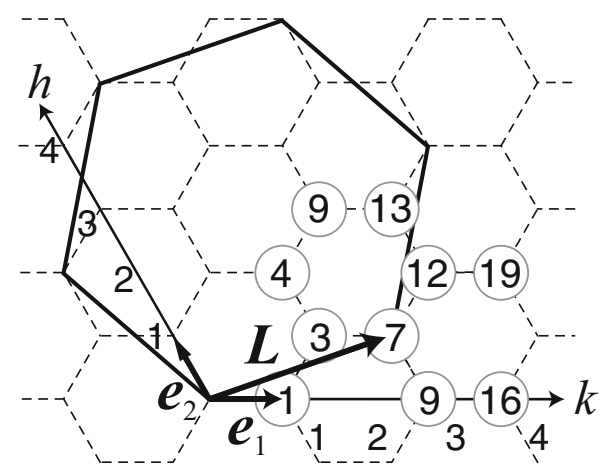

$k^{2}+h k$ and $H=h^{2}+k^{2}-h k: T$ is half the number of lattice points inside a triangle (solid line), while $H$ is the number of dashed hexagons inside a hexagon (solid line). Shown here are $T=13$ with $(3,1)$ and $H=7$ with $(3,1)$ 

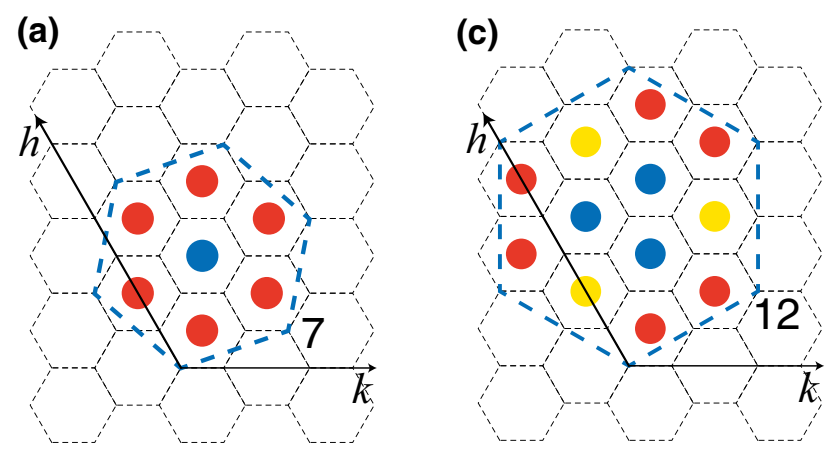

(b)

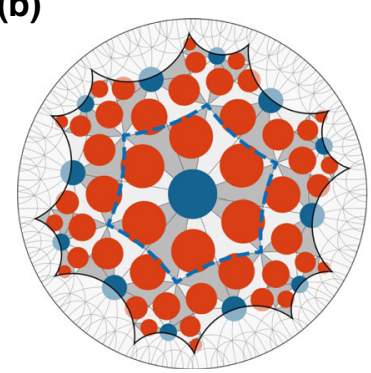

(d)

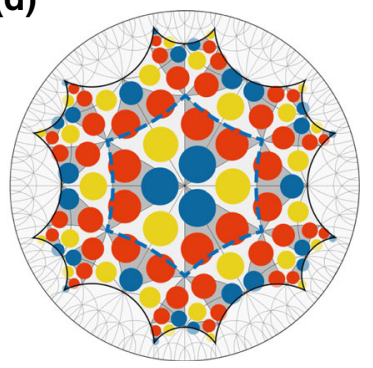

Fig. $4 H$-diagram and a Poincaré disk representing hyperbolic geometry, on which tessellation of $N_{\mathrm{P}}(=8 H)$ spheres is illustrated. a $H$-diagram for $H=7\left(N_{\mathrm{P}}=56\right)$ and $\mathbf{b}$ hyperbolic tiling $\left(3^{6} ; 3^{5} .4\right)$ on the Poincaré disk for (a). c $H$-diagram for $H=12\left(N_{\mathrm{P}}=96\right)$ and d hyperbolic tiling $\left(3^{6} ; 3^{6} ; 3^{5} .4\right)$ tiling on the Poincaré disk for (c). Blue dashed hexagons in (a) and (c) correspond to blue dashed hexagonal regions at the center of (b) and (d), respectively. Then four hexagons connected edge to edge around a vertex of the dashed hexagons are visible on the Poincare disk. The radii of circles on the Poincaré disk are constant in each figure. Figure $4 \mathrm{a}, \mathrm{b}$ displays a system with $N_{\mathrm{P}}=56,(h, k)=(3,1)$ and $H=7$; In $(\mathbf{b})$, blue $\left(3^{6}\right)$ and red $\left(3^{5} .4\right)$ circles correspond to $8 \mathrm{c}$ and $48 \mathrm{i}$ Wyckoff positions of the space group $\operatorname{Pn} \overline{3} n$, respectively, forming a tiling shown in Fig. $2 \mathrm{~g}$. In (d), blue $\left(3^{6}\right)$, yellow $\left(3^{6}\right)$, and red $\left(3^{5} .4\right)$ circles correspond to $24 \mathrm{~g}$, $24 \mathrm{~g}$, and $48 \mathrm{~h}$ of $I \overline{4} 3 \mathrm{~m}$, respectively, forming a tiling shown in Fig. $2 \mathrm{c}$ (Color figure online)

the dodecagonal region is clearly visible in Fig. 4a-d. Blue dashed hexagons in Fig. 4a, c correspond to blue dashed hexagonal regions at the center of Fig. $4 \mathrm{~b}$, d, respectively. On the TPMSs, four hexagonal patches are connected edge to edge around a vertex position of the hexagonal patches making negative curvatures, whose property is rendered on the Poincare disk (Fig. 4b, d). The radii of circles on the Poincare disk are constant in each figure, even though they look smaller as it approaches the circumferences. Figure $4 \mathrm{a}, \mathrm{b}$ displays a system with $N_{\mathrm{P}}=56,(h, k)=(3,1)$ and $H=7$; blue $\left(3^{6}\right)$ and red $\left(3^{5} .4\right)$ circles correspond to $8 \mathrm{c}$ and $48 \mathrm{i}$ Wyckoff positions of the space group $P n \overline{3} n$, constructing a polygonal tiling in Fig. $2 \mathrm{~g}$. Figure $4 \mathrm{c}$, d illustrates a system with $N_{\mathrm{P}}=96,(h, k)=(4,2)$ and $H=12$. Blue $\left(3^{6}\right)$, yellow $\left(3^{6}\right)$, and red $\left(3^{5} .4\right)$ circles correspond to $24 \mathrm{~g}, 24 \mathrm{~g}$, and $48 \mathrm{~h}$ of $I \overline{4} 3 \mathrm{~m}$, forming a polygonal tiling in Fig. 2c, respectively.

\section{Discussion}

We have found that hard spheres on the TPMSs are entropically self-organized when the number of spheres takes some magic numbers $N$. In analogy with the triangulation number for icosahedral viruses, we have proposed the hexagulation number explaining the magic numbers associated with cubic symmetry. With few exceptions ( $H^{*}=3$ class), $N$ is easily evaluated by $H$ in a unified scheme Eq. (3).

From this scheme, we expect P-G-D triplets, which actually holds for $H=4$ and $H=7$. However, the first and the second rows of Table 1 appear to be incomplete. In the first row, $N_{\mathrm{P}}=20$ and $N_{\mathrm{G}}=40$ are shown, but $N_{\mathrm{D}}=80$ is missing; any sign of a transition for $N_{\mathrm{D}}=80$ has not been observed. For $N_{\mathrm{P}}=20$, the distance between two hard spheres is equidistant $(0.3536)$ and a transition has been found. For $N_{\mathrm{G}}=40$, the distance is 0.2795 or 0.3062 forming isosceles triangles, whose $8.7 \%$ difference does not matter to undergo a clear transition [14]. However, for $N_{\mathrm{D}}=80$, the corresponding distance is 0.2165 or 0.2500 , whose difference is too large to induce an Alder transition. This incompleteness of the $\mathrm{P}-\mathrm{G}-\mathrm{D}$ triplet indicates that the property of (almost) equidistance between two hard spheres seems to be indispensable to physical ordering even on the curved background.

In the second row of Table 1, we list $N_{\mathrm{G}}=48$ and $N_{\mathrm{D}}=96$, but not $N_{\mathrm{P}}=24$. The physical arrangement is more complex than we expected; surprisingly, for $N_{\mathrm{P}}=24$ we have had a clear transition, but the unit cell is a doublecell superstructure $(N=192)$ with rhombohedral symmetry. We have seen a transition to a cubic structure in a simulation box with an odd number of the unit cells in a direction; however, the jump in the acceptance ratio is smaller than that of the transition to the double-cell superstructure, implying that the cubic structure is an artifact by the periodic boundary conditions. This exemplifies the difficulty in simulations, and several box sizes should be tested.

On a sphere, complicated arrangements with lower symmetries have been elucidated [40]. Likewise, our ongoing investigation indeed suggests that there exist complicated regular arrangements with lower symmetries even on the TPMSs. Moreover, not only $N_{\mathrm{P}}=24$, but also several unprecedented superstructures for other $N$ have been found. They are beyond the scope of the present paper and will be discussed elsewhere.

Acknowledgments This work was supported by Japan Society for the Promotion of Science (JSPS) Grant-in-Aid for Scientific Research (C) (Numbers $25400431 \& 25400072$ ) \& (B) (Number 16H04037). T. D. is grateful to G. Grason, S. Hyde, R. Kamien, J. Matsuzawa, and A. Schoen for useful comments and stimulating discussions. 
Open Access This article is distributed under the terms of the Creative Commons Attribution 4.0 International License (http://crea tivecommons.org/licenses/by/4.0/), which permits unrestricted use, distribution, and reproduction in any medium, provided you give appropriate credit to the original author(s) and the source, provide a link to the Creative Commons license, and indicate if changes were made.

\section{References}

1. Chaikin PM, Lubensky TC (1995) Principles of condensed matter physics. Cambridge Univ. Press, Cambridge

2. Thomson JJ (1904) On the structure of the atom: an investigation of the stability and periods of oscillation of a number of corpuscles arranged at equal intervals around the circumference of a circle; with application of the results to the theory of atomic structure. Phil Mag 7:237-265

3. Fejes Tóth L (1952) Lagerung in der Ebene auf der Kugel und im Raum. Springer, Berlin

4. Mackay AL, Finney JL, Gotoh K (1977) The closest packing of equal spheres on a spherical surface. Acta Crystallogr Sect a 33:98-100

5. Goldberg MA (1934) Class of multi-symmetric polyhedra. Tohoku Math J 40:226-236

6. Caspar DL, Klug A (1962) Physical principles in the construction of regular viruses. Cold Spring Harbor Symp Quant Biol 27:1-24

7. Zandi R, Reguera D, Bruinsma RF, Gelbart WM, Rudnick J (2004) Origin of icosahedral symmetry in viruses. Proc Natl Acad Sci USA 101:15556-15560

8. Grason GM (2015) Geometry and optimal packing of twisted columns and filaments. Rev Mod Phys 87:401

9. Rubinstein M, Nelson DR (1983) Dense-packed arrays on surfaces of constant negative curvature. Phys Rev B 28:6377-6386

10. Modes CD, Kamien RD (2008) Geometrical frustration in two dimensions: idealizations and realizations of a hard-disk fluid in negative curvature. Phys Rev E 77:041125

11. Irvine WTM, Vitelli V, Chaikin PM (2010) Pleats in crystals on curved surfaces. Nature 468:947-951

12. Zou X, Conradsson T, Klingstedt M, Dadachov MS, O'Keeffe M (2005) A mesoporous germanium oxide with crystalline pore walls and its chiral derivative. Nature 437:716-719

13. Matsushita Y, Hayashida K, Dotera T, Takano A (2011) Kaleidoscopic morphologies from ABC star-shaped terpolymers. J Phys: Condens Matter 23:284111

14. Dotera T, Kimoto M, Matsuzawa J (2012) Hard spheres on the gyroid surface. Interface Focus 2:575-581

15. Mackay AL, Terrones H (1991) Diamond from graphite. Nature 352:762-762

16. Nesper R, Leoni S (2001) On tilings and patterns on hyperbolic surfaces and their relation to structural chemistry. ChemPhysChem 2:413-422

17. Lord EA, Mackay AL, Ranganathan S (2006) New geometries for new materials. Cambridge Univ. Press, Cambridge

18. Ramsden SJ, Robins V, Hyde ST (2009) Three-dimensional Euclidean nets from two-dimensional hyperbolic tilings: kaleidoscopic examples. Acta Cryst A 65:81-108
19. Ramsden SJ, Robins V, Hyde ST, Hungerford S (2005-2009) EPINET: euclidean patterns in non-euclidean tilings. The Australian National University. http://epinet.anu.edu.au/

20. Schoen AH (1970) Infinite periodic minimal surfaces without self-intersections. NASA Technical Note D-5541

21. Charvolin J, Sadoc JF (1987) Periodic systems of frustrated fluid films and bicontinuous cubic structures in liquid crystals. J Physique 48:1559-1569

22. Góźdź W, Hołyst R (1996) From the Plateau problem to periodic minimal surfaces of lipids, surfactants and diblock copolymers. Macromol Theory Simul 5:321-332

23. Hyde S, Andersson S, Larsson K, Blum Z, Landh T, Lidin S, Ninham B (1997) The language of shape. Elsevier, Amsterdam

24. Sadoc JF, Mosseri R (2006) Geometrical frustration. Cambridge Univ. Press, Cambridge

25. Hyde S, O'Keeffe M, Proserpio D (2008) A short history of an elusive yet ubiquitous structure in chemistry, materials, and mathematics. Angew Chem Int Ed 47:7996-8000

26. Nissen HU (1969) Crystal orientation and plate structure in echinoid skeletal units. Science 166:1150-1152

27. Almsherqi ZA, Kohlwein SD, Deng Y (2006) Cubic membranes: a legend beyond the Flatland* of cell membrane organization. J Cell Biol 173:839-844

28. Terasaki O et al (2004) Mesoporous crystals and related nanostructured materials. Elsevier, Amsterdam

29. Dotera $\mathrm{T}$ (2002) Tricontinuous cubic structures in $\mathrm{ABC} / \mathrm{A} / \mathrm{C}$ copolymer and homopolymer blends. Phys Rev Lett 89:205502

30. Dinsmore AD, Hsu MF, Nikolaides MG, Marquez M, Bausch AR, Weitz DA (2002) Colloidosomes: selectively permeable capsules composed of colloidal particles. Science 298:1006-1009

31. Baumgart T, Hess ST, Webb WW (2003) Imaging coexisting fluid domains in biomembrane models coupling curvature and line tension. Nature 425:821-824

32. Herzig EM, White KA, Schofield AB, Poon WCK, Clegg PS (2007) Bicontinuous emulsions stabilized solely by colloidal particles. Nat Mater 6:966-971

33. Subramaniam AB, Abkarian M, Mahadevan L, Stone HA (2005) Colloid science: non-spherical bubbles. Nature 438:930-930

34. Alder BJ, Wainwright TE (1957) Phase transition for a hard sphere system. J Chem Phys 27:1208-1209

35. Frenkel D (1999) Entropy-driven phase transitions. Phys A 263:26-38

36. von Schnering HG, Nesper R (1991) Nodal surfaces of Fourier series: fundamental invariants of structured matter. Z Phys B Condens Matter 83:407-412

37. Gandy PJF, Bardhan S, Mackay AL, Klinowski J (2001) Nodal surface approximations to the P, G, D and I-WP triply periodic minimal surfaces. Chem Phys Lett 336:187-195

38. Wohlgemuth M, Yufa N, Hoffmann J, Thomas EL (2001) Triply periodic bicontinuous cubic microdomain morphologies by symmetries. Macromolecules 34:6083-6089

39. Hyde ST, Ramsden SJ, Robins V (2014) Unification and classification of two-dimensional crystalline patterns using orbifolds. Acta Cryst A 70:319-337

40. Clare BW, Kepert DL (1986) The closest packing of equal circles on a sphere. Proc R Soc Lond A 405:329-344 